\section{El derecho a dar luz sin violencia: una aproximación cuantitativa sobre la violencia obstétrica en Costa Rica}

\author{
Daniela Quesada Coronado y Rafael Román Quirós \\ Universidad de Iberoamérica
}

\section{Resumen}

La violencia obstétrica es un problema de derechos humanos a nivel mundial que afecta a millones de mujeres cada año. Esta investigación analizó desde una perspectiva cuantitativa, correlacional y transversal la relación entre el nivel de percepción de violencia obstétrica en madres costarricenses y diferentes condiciones sociodemográficas y de parto utilizando un instrumento que permitiera medir y clasificar la violencia obstétrica. La recolección de los datos se realizó durante el mes de junio de 2020 a partir de 107 mujeres que dieron a luz por parto vaginal. Los resultados indican que un $49.5 \%$ de las participantes percibió violencia obstétrica ya sea durante el parto, el puerperio o ambos. Se encuentra una correlación negativa entre el nivel de violencia obstétrica percibido y el año en que se dio a luz. No se encuentra que exista una correlación entre el nivel de violencia obstétrica y la edad de la madre al dar a luz o en cuanto al haber tenido la experiencia en zonas urbanas o rurales. Se encuentra además que las manifestaciones más comunes fueron el impedimento para tomar otra posición aparte de la horizontal durante la labor de parto, la imposibilidad de elegir cómo disponer de su placenta y el sufrir más de cinco tactos vaginales.

Palabras clave: Violencia obstétrica, violencia contra la mujer, salud maternal, parto.

\begin{abstract}
Obstetric violence is a global human rights problem that affects millions of women per year. This quantitative, correlational, and cross-sectional research analyzed the association between the perception of obstetric violence levels in Costa Rican mothers and the respective sociodemographic and delivery conditions by using an instrument to measure and classify obstetric violence. Surveys were administered in June 2020 and were conducted on 107 women who gave birth by vaginal delivery. The results indicate that $49.5 \%$ of the participants perceived obstetric violence, either during childbirth, the puerperium, or both. A negative correlation was found between the year after giving birth and the perception of obstetric violence. There is no correlation between obstetric violence level and the age of the mother or whether the birth took place in rural or urban hospitals. It was also found that the most common manifestations were: the impediment to deliver in any position other than horizontal, the refusal to consult about how she wanted to dispose of her placenta, and having more than five vaginal examinations.
\end{abstract}

Keywords: Obstetric violence, violence against women, maternal health, birth. 


\section{Introducción}

La Revolución Industrial en el siglo XIX implicó una gran cantidad de avances tecnológicos, económicos y políticos (Gupta \& Nikodem, 2000). Uno de estos cambios sociales relacionados con la salud pública fue el traslado de los partos de los hogares a los hospitales. Sin embargo, a pesar de que dicho cambio brindó una mayor protección médica a las mujeres en labor de parto (Wooden, 1961), la institucionalización propulsó también ciertas desventajas y cierta propensión hacia los abusos contra la mujer.

Muchas mujeres alrededor del mundo reciben un trato irrespetuoso, ofensivo o negligente durante el parto (Silal et al., 2012; Small et al., 2002; d' Oliveira et al., 2002). Es frecuente, por ejemplo, que durante el primer embarazo de una mujer se realice una episiotomía durante el parto, un procedimiento quirúrgico que amplía la parte inferior de la vagina, el anillo vulvar y el tejido perineal (Sánchez, 2012, citado por Ballesteros-Meseguer et al., 2016). A pesar de que se trata de uno de los procedimientos más utilizados en obstetricia, la evidencia científica no apoya el procedimiento como una práctica de rutina (Carroli \& Mignini, 2009). Es frecuente que el mismo sea realizado sin el consentimiento de la mujer que se encuentra en labor de parto (Rahim \& Rafiq, 2018), que en ocasiones se lleve a cabo sin el uso de anestesia (Bowser \& Hill, 2010) o que el procedimiento pueda tener como consecuencia permanente la pérdida en el control de los esfínteres (Lappen \& Gosset, 2010).

Otro ejemplo se encuentra en el caso de las cesáreas, un procedimiento que si bien puede prevenir eficazmente la morbilidad y mortalidad materna y perinatal en ciertas circunstancias, puede también implicar un problema si el mismo no se encuentra médicamente justificado; esto porque la cesárea en sí misma implica un aumento de morbilidad en las mujeres que pasan por la experiencia en comparación con un parto natural (Glazener et al., 1995) a la vez que no se conocen beneficios para aquellas que pasan por el procedimiento sin ser requerido (Pope, 2017). Se trata de un riesgo que según un metaanálisis y revisión sistemática de Sobhy et al. (2019) no ha disminuido en los últimos 30 años. Si bien la comunidad internacional ha definido desde 1985 que la tasa ideal de cesáreas se sitúa entre el 10 y el 15\% (Betrán et al., 2016), América Latina es al día de hoy, la región en donde se concentra la mayor tasa de cesáreas del mundo con varios países por encima del $40 \%$, lo que evidentemente plantea dudas en cuanto a la justificación del grueso de estas intervenciones (Sadler et al., 2016). Es importante aclarar que la violación a los derechos de las mujeres durante sus procesos de parto constituye una violación de derechos humanos (Al Adib et al., 2017; Khosla et al., 2016) y que en este sentido, el derecho de la mujer embarazada a negarse a este procedimiento particular por ejemplo, debe ser incondicional, en el tanto existe una prioridad del mismo por sobre las preferencias de los médicos tratantes.

Es en estos casos que se hace referencia a la violencia obstétrica, un concepto que abarca diferentes formas de violencia que son ejercidas por el profesional de salud sobre las mujeres durante sus procesos reproductivos y las cuales se presentan principalmente (más no exclusivamente) a través de un trato deshumanizado hacia la mujer embarazada, en la patologización innecesaria de los procesos reproductivos y en distintas manifestaciones que 
resultan amenazantes para la mujer en relación con la atención que recibe por parte del centro y de los profesionales en salud (Belli, 2013).

Los ejemplos de abuso durante el parto van desde proveedores de servicios que pellizcan a las mujeres hasta el abuso sexual. Bowser y Hill (2010) mencionan diferentes ejemplos de abuso físico obstétrico como el ser abofeteada, el estar restringida o atada durante el parto, suturas posparto de desgarros vaginales sin el uso de anestesia, o ser sometida a empujones del abdomen para que mediante el uso de la fuerza física excesiva el niño sea expulsado fuera del útero.

Los mismos autores señalan de igual manera situaciones en las que el trato resulta irrespetuoso y abusivo durante el parto; situaciones humillantes que implican el abuso verbal, la falta de consentimiento ante diferentes intervenciones (incluida la esterilización), la falta de confidencialidad, la negativa a dar medicamentos para el dolor cuando está indicado, o violaciones a la privacidad. Desde una perspectiva de negligencia, autores como d'Oliveira et al. (2002) indican que la situación de tener mujeres que dan a luz sin atención médica en los establecimientos de salud resulta también común.

Las situaciones anteriores son sólo algunos ejemplos que evidencian la necesidad e importancia que tiene el garantizar condiciones seguras tanto físicas como psicológicas para las madres gestantes; sin embargo existe también un riesgo para el neonato. Piénsese por ejemplo en el caso de una mujer que habiendo llevado un embarazo saludable, le es recetada oxitocina para una mejor labor de parto a la vez que no se le provee el debido monitoreo; una situación que de igual manera podría ocasionar un daño al feto (Herrera, 2016).

Resulta a la vez preocupante que a pesar de todo lo anterior, la tasa de intervenciones obstétricas no justificadas médicamente haya aumentado en países de ingresos medios y altos sin que tampoco dichas prácticas contribuyan en alguna medida importante a mejoras en la mortalidad y morbilidad perinatal y materna (Zeitlin et al., 2013). Existe una preocupación creciente desde el año 1979 (hace más de 40 años) en cuanto al maltrato y las faltas de respeto como un problema importante de discriminación contra la mujer en labor de parto (Convención sobre la eliminación de todas las formas de discriminación contra la mujer, 1979) y desde 1985, la Organización Mundial de la Salud y la Organización Panamericana de la Salud advertían respecto a diferentes preocupaciones en torno al parto y las tecnologías utilizadas (la Declaración de Fortaleza) urgiendo tanto a administradores como al personal de salud a hacer una revisión de los protocolos concernientes a las prácticas médicas que ponían en riesgo la autonomía y la perspectiva de la mujer en la toma de decisiones durante el proceso (Organización Mundial de la Salud, 1988). Lo cierto es que a pesar de las pronunciaciones de diferentes organizaciones internacionales durante décadas, un número cada vez mayor de investigaciones sobre la violencia obstétrica alrededor del mundo plantean un panorama desalentador (White Ribbon Alliance, 2017).

La evidencia sugiere que el maltrato durante el parto, incluido el abuso físico, verbal, la discriminación, los procedimientos no consentidos y el cuidado sin apoyo continúan estando presentes de manera alarmante alrededor del mundo (Bohren et al., 2015). Un estudio realizado en cuatro países africanos (Ghana, Guinea, Myanmar y Nigeria) e impulsado por la Organización de las Naciones Unidas, indica que más de un tercio de las mujeres sufren violencia obstétrica a la hora de dar a luz (Bohren et al., 2015). En Venezuela el 79.5\% de las mujeres percibieron alguna manifestación de violencia obstétrica durante su proceso de parto y puerperio (Terán et al., 2013), 
en Italia un 21.2\% (Ravaldi et al., 2018) y en España un 38.3\% (Mena-Tudela et al., 2020). Aunado a los porcentajes anteriores, Kukura (2018) indica que para el 2017 en Estados Unidos no había un estudio exhaustivo sobre el tema de la violencia obstétrica, lo que significa que no existe una estimación de su prevalencia en la literatura investigativa de este país, al menos hasta el año 2018.

En Costa Rica, según la Encuesta Estado de la Mujer, Niñez y Adolescencia; llevada a cabo en el año 2018 por el Instituto Nacional de Estadística y Censos (INEC) y en colaboración con el Ministerio de Salud (MS), el 31.6\% de las mujeres con hijos nacidos por parto vaginal en los últimos dos años sufrieron violencia obstétrica entre el 2016 y el 2018. La negativa a consultar la aplicación de procedimientos médicos o la administración de medicamentos con las pacientes, consistió en la forma de violencia más común (Instituto Nacional de Estadística y Censos, 2019).

En cumplimiento con un informe emitido por la Defensoría de los Habitantes, la Caja Costarricense del Seguro Social ordenó proceder con la apertura de procedimientos administrativos disciplinarios cuando existan casos de violencia obstétrica en los servicios de maternidad (Defensoría de los Habitantes, 2019). Adicionalmente se aprobó en febrero del año 2020 la ley que reforma el artículo 12 de la Ley General de Salud para asegurar una intervención médica respetuosa de las mujeres embarazadas durante todo el proceso de parto. Se postulan en dicha ley una serie de principios para asegurar el bienestar de la mujer embarazada y eliminar las intervenciones innecesarias en los centros de salud costarricenses (Instituto Nacional de la Mujer, 2020). Dichos principios incluyen el derecho a recibir información clara antes, durante y después del parto en cuanto a tratamientos, diagnósticos, pronósticos o posibles intervenciones médicas; además, el derecho a parir de manera natural siempre y cuando no exista un riesgo de salud para la madre o para el niño o niña por nacer, el derecho de la madre a estar con su bebé después del parto, y el derecho a tener compañía de una persona designada por la madre durante el parto y postparto (Díaz, 2020). Actualmente el país se encuentra a la espera de la aprobación del proyecto de ley 19537: la ley para proteger a la mujer embarazada y sancionar la violencia obstétrica (Fuentes-Rodríguez, 2020).

Si se consideran los porcentajes de incidencia de Violencia Obstétrica vistos anteriormente para otras naciones, Costa Rica en comparación contaría con un elevado porcentaje de ocurrencia que se ubica por encima de Italia, Ghana, Guinea, Nigeria, Myanmar o España, por ejemplo. Sin embargo, es importante aclarar que las comparaciones en este sentido resultan difíciles de establecer, en el tanto no existe un consenso internacional en cuanto a la definición de VO, y su medición (Bohren et al., 2015; Mena-Tudela et al., 2020; Organización Mundial de la Salud, 2014; Bowser \& Hill, 2010). A pesar de esto, resulta más que evidente que los porcentajes encontrados resultan alarmantes, sobre todo si consideramos que se trata de manifestaciones de violencia que atentan contra los derechos fundamentales de la población.

En cuanto a diferentes condiciones socioeconómicas que han sido relacionadas en la literatura con una mayor propensión a ser objeto de VO, se encuentra el vivir en un lugar con difícil acceso a servicios de salud, el hablar alguna lengua indígena o dialecto, el ser menor de edad, el vivir en un contexto de pobreza y los bajos o nulos niveles de educación (Organización Mundial de la Salud, 2019). Un estudio liderado por la Organización Mundial de la Salud y otras 
organizaciones mundiales descubrió que las posibilidades de ser víctimas de violencia obstétrica son mayores en mujeres jóvenes con niveles bajos de educación y que su ocurrencia es mayor entre los treinta minutos antes del parto y los quince minutos después del mismo. El aumento del riesgo durante este período temporal podría deberse a que es más probable que el personal de salud esté presente en el momento del nacimiento, o también debido a estresores que influyen en el comportamiento del personal del área de salud (Bohren et al., 2015).

En el caso particular de Costa Rica y según el Instituto Nacional de Estadística y Censos (2019) no se encuentran diferencias importantes en VO en labor de parto (por vía vaginal) entre el área rural y urbana $(34.1 \%$ y $25.7 \%$ ); en donde, por ejemplo, la provincia de Cartago posee $39.6 \%$ de casos. Llama la atención que las mujeres del último quintil (las de mayor nivel adquisitivo) experimentaran en un $15.5 \%$ VO (por vía vaginal) respecto al 35,6\% y al $45.8 \%$ del primer y segundo quintil (y de menor nivel adquisitivo).

Dentro de las investigaciones realizadas en Costa Rica sobre el tema, Arguedas (2014) señala una paternidad médica en referencia a una relación de dominancia por parte del personal médico hacia la mujer embarazada, en la que se le impide a esta ejercer su autonomía y libertad en la toma de decisiones. Además, se describe como un proceso rutinario el acelerar la labor de parto (en ocasiones administrando oxitocina), mujeres informadas a medias y sin poder tomar decisiones en su proceso de dar a luz, así como evidencias de xenofobia y racismo.

En cuanto a la investigación en general realizada en Costa Rica sobre el tema, se encontraron primordialmente investigaciones cualitativas (Arguedas, 2014; Rodríguez \& Vargas, 2017), dos esfuerzos nacionales de tipo cuantitativo (por parte del Ministerio de Salud en el 2011 y por parte del INEC en el 2018; ambas con auspicio de UNICEF) y una investigación en el 2020 a partir de los datos generados por ambos esfuerzos nacionales (Fuentes-Rodríguez, 2020). Es decir, a partir de la búsqueda realizada en el repositorio nacional (Kimuk) y en sus repositorios específicos, así como en bases de datos latinoamericanas de acceso abierto, se encuentra que la investigación académica de tipo cuantitativo en cuanto al tema resulta sumamente escasa en el país.

En este sentido, la presente investigación representa un acercamiento cuantitativo al tema que pretende explorar la utilización de un instrumento que no sólo parta de un concepto más amplio de $\mathrm{VO}$ en partos naturales que el utilizado por las autoridades de salud, sino que además pueda permitir entender la problemática más allá de lo descriptivo. La investigación tiene el objetivo de comparar los niveles de violencia obstétrica en madres costarricenses en cuanto a diferentes condiciones sociodemográficas y de parto como lo son el grado educativo de las mismas, el año en el que dieron a luz (se les pidió referirse a su último embarazo en el caso de haber tenido varios), la edad de la madre al parir, si el parto sucedió en un hospital rural o del Gran Área Metropolitana o si se trataba de un hospital privado o público (de la Caja Costarricense del Seguro Social). Se espera de esta forma contribuir al proceso de encontrar potenciales factores de riesgo y protección en torno a la violencia obstétrica en Costa Rica, contribuyendo a políticas públicas efectivas que permitan mejorar aspectos de prevención y bienestar general en mujeres en labor de parto y posterior al mismo, en los que por sus distintas condiciones se encuentra en un mayor estado de vulnerabilidad. 


\section{Método}

\section{Participantes}

Se contó con la participación de 107 mujeres costarricenses que dieron a luz por vía natural dentro del territorio nacional (es decir, no se tomaron en cuenta aquellas que pasaron por cesáreas en el nacimiento de su(s) último(s) hijo(s). A las participantes se les solicitó referirse específicamente a su parto más reciente en caso de haber tenido varios. El consentimiento informado fue agregado en el instrumento de recolección de datos. Todas las participantes presentaron anuencia en formar parte de la investigación mediante la lectura y aceptación del mismo, donde se expusieron los objetivos de la investigación, riesgos, beneficios y se aclara el derecho al resguardo de los datos, anonimato y confidencialidad de la información ofrecida. Es importante destacar que la recolección de datos fue por vía virtual debido a la situación nacional con la pandemia relacionada con la COVID-19.

\section{Instrumento}

Para la conformación del cuestionario se incluyeron una serie de ítems sociodemográficos iniciales seguidos por el instrumento confeccionado por Risco (2019) que busca identificar situaciones de violencia obstétrica. La validación del instrumento se hizo mediante jueces, y la autora reporta un Alfa de Cronbach de 0.889 en una prueba piloto. Este último fue desarrollado para la investigación titulada "Violencia obstétrica durante la atención del parto, puerperio y la percepción de la usuaria". El instrumento original cuenta con cuatro secciones, de las cuales se dejó por fuera la primera y cuarta, debido al hecho de que la primera parte fue sustituida por ítems sociodemográficos de mayor interés para esta investigación, y debido a que la última parte referida al personal médico por el cual fueron atendidas las participantes podría no poderse precisar debido a que algunas encuestadas tuvieron sus partos años atrás (en ocasiones incluso décadas). En este sentido, las secciones retiradas (I y IV) no cuentan con ítems que contribuyan al puntaje del nivel de VO, puesto que los mismos consisten en condiciones y categorías del servicio obstétrico recibido.

En su aplicación, el instrumento contó con 21 preguntas divididas en atención durante el parto (ítems del 1 al 15) y atención durante el puerperio (ítems del 16 al 21). Cada ítem recibe el puntaje de la siguiente manera: siempre (2 puntos), a veces (1 punto), nunca (0 puntos), SI (2 puntos), NO (0 puntos), y no le hicieron el procedimiento (0 puntos). Los resultados se procesan de la siguiente manera: En la II Sección si la suma de los resultados se encuentra entre 0 y 9 se considera que la usuaria no percibió VO; los casos que se encuentran entre 10 y 30 puntos se consideran casos positivos por VO durante el parto. En la III Sección si la suma de los resultados es superior a 7 (hasta 12), se considera que la usuaria experimentó VO durante el puerperio. Adicionalmente los ítems que indicarían VO de tipo psicológico serían los ítems del 1 al 8 de la 
segunda sección, así como el ítem 16 y 18. Los ítems que indicarían VO de tipo física son aquellos que se encuentran entre el 9 y el 15, así como entre el 19 y el 21 (Risco, 2019).

\section{Procedimientos de recolección}

Luego de confeccionado el cuestionario virtual utilizando la plataforma Google Forms y se utilizó el link o enlace provisto por la plataforma para distribuir el cuestionario por medios electrónicos, en particular por correo electrónico y Whatsapp a personas conocidas por los investigadores y utilizando el método Snowball. En particular dentro del Whatsapp se contó con la participación de mujeres miembros del grupo de mujeres "Somos Tribu" (un grupo dedicado al crecimiento de mujeres en temas de maternidad y crianza). Dentro del cuestionario se incluyó el consentimiento informado que enfatizaba los criterios de exclusión y un ítem en el que expresamente se debía confirmar (haciendo clic) en cuanto a haber leído la totalidad del mismo estando de acuerdo con participar.

\section{Procedimientos de análisis}

Se realizaron análisis descriptivos e inferenciales de las variables relacionadas a la muestra en relación con la percepción de la violencia obstétrica utilizando el Programa Estadístico SPSS, versión 23 (SPSS Inc., Chicago, Illinois, USA).

\section{Resultados}

En la muestra hay un total de 107 mujeres participantes que cumplen con los criterios de inclusión y que tuvieron su último hijo entre 1982 y el 2020. Las edades de las mujeres participantes fueron entre 19 y 74 años $(\mathrm{M}=36.53, \mathrm{DE}=10.77)$. Un $72 \%$ de la muestra cuenta con algún grado de educación universitaria (un $37.4 \%$ con el grado de licenciatura). Casi la mitad de las mujeres encuestadas (el 50.5\%) tuvo su último hijo entre el 2013 y el 2020, el 31.8\% de la muestra dio a luz entre el 2018 y el 2020; la mayoría (el 14.8\%) durante el 2019. La edad promedio en que las participantes dieron a luz a su(s) último(s) hijo(s) es de 27.3 (DE=5.89). La edad mínima en que una participante dio a luz fue a los 17 y la mayor a los 39.

Un $90.7 \%$ de la muestra tuvo a su último hijo en un hospital público (de la Caja Costarricense del Seguro Social). Esto quiere decir que solamente 10 mujeres dieron a luz en un hospital privado (9.3\%), por lo que este segmento de la muestra no resultaría no representativo para efectos de análisis. Un 57.9\% dio a luz en un hospital del Gran Área Metropolitana (GAM), mientras que un $38.3 \%$ dio a luz en un hospital ubicado en una zona rural. 


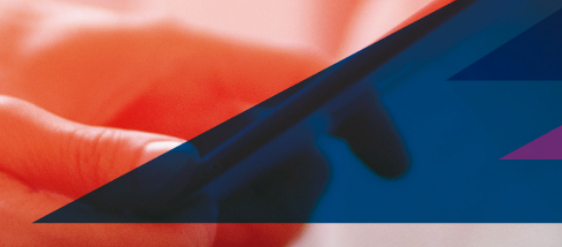

PsicoInnova, 5(1), 2021, 12-26

Los resultados indican que un $46.7 \%$ de las mujeres sufrieron VO durante su labor de parto, mientras que un $23.4 \%$ indican haberlo experimentado durante el puerperio. En total, un $49.5 \%$ de las mujeres habrían sufrido VO ya sea durante el parto, o el puerperio o en ambas circunstancias bajo el instrumento diseñado por Risco (2019). La media de VO total fue de 13.96 (DE=9.05), con un promedio de 7.36 para la VO psicológica y de 6.60 para la VO física.

En cuanto a los hallazgos inferenciales, se encontró una correlación negativa entre el año de los partos y los niveles de violencia obstétrica, lo que significa que el dar a luz en años más recientes se relacionaría con menores niveles de VO en general $(r=-.25, \mathrm{p}=.009)$, en particular, existe una relación en cuanto a menores niveles de $\mathrm{VO}$ de tipo físico en partos más recientes $(r=-.29, \mathrm{p}=.003)$.

No se encontró una correlación significativa entre la edad de la madre al parir y otros indicadores de violencia obstétrica $(r=-.017, \mathrm{p}=.861)$, así como tampoco se encontró una relación entre violencia obstétrica y el nivel educativo de la mujer entrevistada $(r=.03, p=.69)$ (Vdr Tabla $1)$.

Tabla 1. Estadística descriptiva y correlaciones para las variables de estudio

\begin{tabular}{lcccccccccc}
\hline \multicolumn{1}{c}{ Variable } & $n$ & $M$ & $S D$ & 1 & 2 & 3 & 4 & 5 & 6 & 7 \\
\hline 1. Violencia obstétrica & 107 & 13.96 & 9.05 & - & & & & & \\
2. Edad actual & 107 & 36.53 & 10.77 & $.24^{*}$ & - & & & & \\
3. Grado académico actual & 107 & 4.14 & 1.26 & .03 & .16 & - & & & \\
$\begin{array}{l}\text { 4. Edad en que se dio a luz al } \\
\text { último hijo }\end{array}$ & 107 & 27.30 & 5.89 & -.02 & $.53^{* *}$ & $.34^{* *}$ & - & & \\
$\begin{array}{l}\text { 5. Año en que se dio a luz } \\
\text { 6. Violencia obstétrica durante el } \\
\text { parto }\end{array}$ & 107 & 2010.27 & 9.52 & $-.25^{* *}$ & $-.81^{* *}$ & .08 & -.01 & - & \\
$\begin{array}{l}\text { 7. Violencia obstétrica durante el } \\
\text { puerperio }\end{array}$ & 107 & 9.51 & 6.65 & $.98^{* *}$ & $.25^{*}$ & .03 & -.03 & $-.26^{* *}$ & - \\
${ }^{*} p<.05 .^{* *} p<.01$. & 107 & 4.45 & 2.95 & $.87^{* *}$ & .18 & .03 & .01 & -.18 & $.74^{* *}-$ \\
\hline
\end{tabular}

Al realizar pruebas $\mathrm{T}$ para muestras independientes, no se encuentraron diferencias significativas entre las áreas rurales y urbanas $\mathrm{t}(101)=.59, \mathrm{p}=.555$. 
Únicamente un $1.9 \%$ de las participantes ( 2 personas) reportaron no haber percibido manifestación alguna de violencia obstétrica. Sin resultar estadísticamente representativas, ambas dieron a luz en hospital de carácter privado en el año 1999 y el 2020.

Por último, las tres formas más comunes de violencia obstétrica entre las participantes fueron el impedimento para tomar otra posición aparte de la horizontal durante su labor de parto, la negativa a consultar sobre cómo deseaban disponer de su placenta y la realización de más de cinco tactos vaginales.

\section{Discusión}

Los resultados obtenidos indican que el porcentaje de mujeres que de una u otra forma han experimentado VO durante sus procesos de parto vaginal (durante y después del mismo) por parte del personal médico es del $49.5 \%$ utilizando el instrumento desarrollado por Risco (2019). Inmediatamente salta a la memoria el $31.6 \%$ reportado por el estudio realizado por el INEC y por el Ministerio de Salud en el 2018 ante las experiencias de VO sufridas por mujeres que dieron a luz entre el 2016 y el 2018 por vía vaginal. Dicho estudio incluye únicamente 9 formas de VO en su cuestionario y otros 6 aspectos que componen lo que en el informe se denomina violencia obstétrica ampliada (Fuentes-Rodríguez, 2021), mientras que el instrumento utilizado para la presente investigación incluye 21 aspectos (20 de los cuales fueron aplicados debido a un error de digitación); lo cual podría explicar la diferencia en el porcentaje obtenido. Al respecto, a pesar de que no existe un consenso mundial sobre la definición de VO, existen manifestaciones que deberían ser incluidas en los instrumentos aplicados a nivel nacional para tener un panorama más preciso y detallado sobre la realidad. En este sentido se destaca la importancia de la validación de un instrumento para la medición de la $\mathrm{VO}$ a nivel nacional.

Por otro lado, los resultados indican una correlación negativa entre el año de haber dado a luz y el nivel de violencia obstétrica percibido; es decir que existe una relación entre la antigüedad del parto y mayores niveles de violencia obstétrica. Esto podría interpretarse como un avance a lo largo de los años en materia de un trato digno y respetuoso hacia las mujeres durante sus labores de parto. No obstante, los resultados obtenidos por el INEC y el Ministerio de Salud indican que tomando en cuenta tanto los partos por vía vaginal como cesárea, el número asciende a un 58.2\% entre el 2016 y el 2018; un dato que resulta alarmante en vista de su magnitud, pues se trata de 135,281 mujeres en un lapso de dos años (INEC, 2019). Lo anterior resulta cuando menos preocupante al considerar que distintas organizaciones mundiales de las cuales Costa Rica es un estado miembro han estado desde hace más de 40 años abogando por una mejora en la calidad y en el respeto de los derechos de las mujeres en diferentes ámbitos (Organización de las Naciones Unidas y la Organización de Estados Americanos por citar dos ejemplos). Es importante agregar, además, que en el estudio publicado hace dos años (INEC, 2019) es la primera vez que se genera información estadística que permite plantear la gravedad de la situación con respecto al tema (Fuentes-Rodríguez, 2021). 
Es por esto que es imperante rescatar que si bien Costa Rica está avanzando en cuanto a ajustes en la legislación y la revisión y creación de procedimientos administrativos y disciplinarios para minimizar la violencia obstétrica (Díaz, 2020), el país se encuentra sumamente atrasado en cuanto a la protección de los derechos humanos de las mujeres en materia obstétrica y dichos ajustes se dan adicionalmente y de manera reciente a instancia de la Defensoría de los Habitantes.

El hecho también de que la mayoría de las expresiones más comunes de violencia obstétrica estén relacionadas con violencia física preocupa. Más de cinco tactos vaginales a una mujer es un abuso físico y negligencia por parte del personal médico para respetar su cuerpo, intimidad y privacidad. Aunado a esto, el no permitir a las mujeres incorporarse en otra posición que no sea la horizontal refuerza la postura expresada por Arguedas (2014), en la que se indica que en Costa Rica existe una paternidad médica durante el parto y confirma que las mujeres están a merced del médico y la comodidad de este para recibir al niño o niña. La ambientación hospitalaria y las acciones del personal de la salud deben estar siempre pensadas y ejecutadas exclusivamente en pos del bienestar tanto del recién nacido como el de la madre.

Es importante destacar además que los factores del instrumento utilizado que se catalogan como violencia obstétrica no tienen que ver con acceso tecnológico ni infraestructura sino con el servicio proporcionado por los profesionales de la salud. La gran mayoría de las mujeres participantes en este estudio dieron a luz en un hospital de carácter público, esto es, en un hospital de la Caja Costarricense del Seguro Social, por lo tanto, es de suma importancia a nivel nacional la capacitación, creación de procedimientos que se revisen continuamente, evaluación del personal y del servicio de atención como parte de un esfuerzo integral para erradicar las manifestaciones de VO.

Si bien no se encontró relación entre el nivel educativo de las madres y la percepción de VO; lo verdaderamente importante no sería tanto el nivel educativo actual, sino el nivel educativo que tenían las mismas en el momento de dar a luz, dato con el cual no se cuenta. De igual forma, al no haber participación de menores de edad dentro del estudio, no se pudo determinar si este factor podría incrementar la posibilidad de ser objeto de VO. Por último, es importante investigar el tema con mujeres que hayan dado a luz en hospitales de carácter privado, así como con mujeres de diferentes etnias y condiciones socioeconómicas que diferentes investigaciones cualitativas en el país han señalado como un aspecto a tomar en cuenta. 


\section{Referencias}

Al Adib Mendiri, M., Ibáñez Bernáldez, M., Casado Blanco, M., y Santos Redondo, P. (2017). La violencia obstétrica: un fenómeno vinculado a la violación de los derechos elementales de la mujer. Medicina Legal de Costa Rica, 34(1), 104-111. http://www.scielo.sa.cr/scielo.php?pid=S1409-00152017000100104\&script=sci_arttext

Alkema, L., Chou, D., Hogan, D., Zhang, S., Moller, A. B., Gemmill, A., Ma Fat, D., Boerma, T.,Termmerman, M., Mathers, C., y Say, L. (2016). Global, regional, and national levels and trends in maternal mortality between 1990 and 2015, with scenario-based projections to 2030: a systematic analysis by the UN Maternal Mortality Estimation Inter-Agency Group. The Lancet, 387(10017), 462-474. https://doi.org/10.1016/S0140-6736(15)008387

Arguedas, G. (2014). La violencia obstétrica: propuesta conceptual a partir de la experiencia costarricense. Cuadernos Inter.ca mbio sobre Centroamérica y el Caribe, 11(1), 145-169. DOI 10.15517/C.A..V11I1.14238

Ballesteros-Meseguer, C., Carrillo-García, C., Meseguer-de-Pedro, M., Canteras-Jordana, M., Martínez-Roche, M. (2016). La episiotomía y su relación con distintas variables clínicas que influyen en su realización. Revista Latinoamericana de Enfermagem, 24, 1-6. $10.1590 / 1518-8345.0334 .2686$

Belli, L. F. (2013). La violencia obstétrica: otra forma de violación a los derechos humanos. Revista Red Bioética UNESCO, 1(7). Recuperado de http://www.unesco.org/new/fileadmin/MULTIMEDIA/FIELD/Montevideo/images/Revis taRedBioeticaA4V1N7-2013.pdf

Betran, A. P., Torloni, M. R., Zhang, J. J., Gülmezoglu, A. M., \& WHO Working Group on Caesarean Section (2016). WHO Statement on Caesarean Section Rates. BJOG : an international journal of obstetrics and gynaecology, 123(5), 667-670. https://doi.org/10.1111/1471-0528.13526

Bohren, M. A., Vogel, J. P., Hunter, E. C., Lutsiv, O., Makh, S. K., Souza, J. P., Aguiar, C., Saraiva Coneglian, F., Diniz, A. L., Tunçalp, Ö., Javadi, D., Oladapo, O. T., Khosla, R., Hindin, M. J., \& Gülmezoglu, A. M. (2015). The Mistreatment of Women during Childbirth in Health Facilities Globally: A Mixed-Methods Systematic Review. PLoS medicine, 12(6), e1001847. https://doi.org/10.1371/journal.pmed.1001847

Bowser, D., y Hill, K. (2010). Exploring evidence for disrespect and abuse in facility-based childbirth. Boston: USAID-TRAction Project, Harvard School of Public Health.

Carroli, G., y Mignini, L. (2009). Episiotomy for vaginal birth. The Cochrane database of systematic reviews, (1), CD000081. https://doi.org/10.1002/14651858.CD000081.pub2

Convención sobre la eliminación de todas las formas de discriminación contra la mujer, 18 de diciembre, 1979. https://www.ohchr.org/sp/professionalinterest/pages/cedaw.aspx 
Defensoría de los Habitantes (2019). Abrirán procedimientos disciplinarios ante denuncias por violencia

obstétrica. http://www.dhr.go.cr/informacion_relevante/comunicados/2019/abril/17_viol encia_obstetrica.pdf

Díaz, N. (2020, 14 de febrero). Mujeres embarazadas tienen ocho nuevos derechos de salud: ¿cuáles son y qué protegen? Semanario Universidad. Recuperado de https://semanariouniversidad.com/pais/mujeres-embarazadas-tienen-ocho-nuevosderechos-de-salud-cuales-son-y-que-protegen/

d'Oliveira, A. F. P. L., Diniz, S. G., y Schraiber, L. B. (2002). Violence against women in healthcare institutions: an emerging problem. The Lancet, 359(9318), 1681-1685. DOI: 10.1016/S0140-6736(02)08592-6

Fuentes-Rodríguez, E. (2021). Violencia obstétrica en Costa Rica desde la evidencia estadística: Retos para la gerencia de la salud y la tutela de los derechos reproductivos. Fondo de Población de Naciones Unidas https://costarica.unfpa.org/sites/default/files/pub-pdf/violencia-obstetrica-encosta_rica.pdf

Fuentes-Rodríguez, E. (2020). Violencia obstétrica y su relación con determinantes sociales de la salud a partir de evidencia estadística en Costa Rica (2009-2011 y 2016-2018). [Tesis inédita de Posgrado en Salud Pública, Universidad de Costa Rica]. http://www.kerwa.ucr.ac.cr/bitstream/handle/10669/81289/Eugenio\%20Fuentes\%20Rodr $\%$ c3\%adguez $\% 20$ Salud\%20P\%c3\%bablica\%20TFG.pdf?sequence=1\&isAllowed=y

Glazener, C. M., Abdalla, M., Stroud, P., Naji, S., Templeton, A., y Russell, I. T. (1995). Postnatal maternal morbidity: extent, causes, prevention and treatment. British journal of obstetrics and gynaecology, 102(4), 282-287. https://doi.org/10.1111/j.1471-0528.1995.tb09132.x

Gupta, J., y Nikodem, C. (2000). Maternal Posture in Labour. European Journal of Obstetrics \& Gynecology and Reproductive Biology, 92(2), 273-277. doi: https://doi.org/10.1016/S0301-2115(99)00272-9

Herrera, C. (2016) Obstetric violence: a new framework for identifying challenges to maternal healthcare in Argentina, Reproductive Health Matters, 24:47, 65-73. 10.1016/j.rhm.2016.05.001

Instituto Nacional de Estadísticas y Censos. (agosto de 2019). Encuesta de Mujer, Niñez y Adolescencia 2018 [archivo de Excel] [Salud reproductiva y materna] https://www.inec.cr/encuestas/encuesta-mujeres-ninez-adolescencia

Instituto Nacional de la Mujer. (2020, 14 de febrero). INAMU celebra aprobación de ley contra la violencia obstétrica. https://www.inamu.go.cr/web/inamu/inamu-celebra-aprobacion-deley-contra-la-violencia-obstetrica

Khosla, R., Zampas, C., Vogel, J. P., Bohren, M. A., Roseman, M., y Erdman, J. N. (2016). International Human Rights and the Mistreatment of Women During Childbirth. Health and human rights, 18(2), 131-143 https://www.ncbi.nlm.nih.gov/pmc/articles/PMC5394989/ 
Kukura, E. (2018). Obstetric violence. Georgetown Law Journal. 106, 721. https://heinonline.org/HOL/LandingPage?handle=hein.journals/glj106\&div=22\&id=\&pa ge=Naciones Unidas (1993). Declaración sobre la Eliminación de la Violencia contra la Mujer. Resolución de la Asamblea General 48/104, de 20 de diciembre de 1993. Recuperado de https://www.servindi.org/pdf/DecEliminacionViolenciaMujer.pdf

Lappen, J. R. y Gosset, D. R. (2010). Changes in episiotomy practice: evidence-based medicine in action. Expert review of obstetrics and ginecology, 5(3), 301-309. https://doi.org/10.1586/eog.10.21

Mena-Tudela, D., Iglesias-Casás, S., González-Chordá, V. M., Cervera-Gasch, Á., Andreu-Pejó, L., y Valero-Chilleron, M. J. (2020). Obstetric Violence in Spain (Part I): Women's Perception and Interterritorial Differences. International journal of environmental research and public health, 17(21), 7726. https://doi.org/10.3390/ijerph17217726

Organización Mundial de la Salud (2019). Mortalidad Materna. Recuperado de https://www.who.int/es/news-room/fact-sheets/detail/maternal-mortality

Organización Mundial de la Salud (2014). Prevención y erradicación de la falta de respeto y el maltrato durante la atención del parto en centros de salud. Recuperado de https://apps.who.int/iris/bitstream/handle/10665/134590/WHO_RHR_14.23_spa.pdf;jsess ionid=A5359FA304A4631F1756ECC9D1BB5BE5? sequence=1

Organización Mundial de la Salud. (1988). WHO Joint Interregional Conference on Appropriate Technology for Birth: Fortaleza, Brazil [Reporte abreviado]. Australian College of Midwives Incorporated Journal, 1(2), 18-19. https://doi.org/10.1016/S1031170X(88)80047-3

Organización de Estados Americanos (OEA). (1994). Inter-American Convention on the Prevention, Punishment and Eradication of Violence against Women ("Convention of Belem do Para"), 9 June 1994. Recuperado de https://www.oas.org/en/mesecvi/docs/BelemDoPara-ENGLISH.pdf

Pope T. M. (2017). Legal Briefing: Unwanted Cesareans and Obstetric Violence. The Journal of clinical ethics, 28(2), 163-173.

Rahim, K. A. y Rafiq, N. (2018). Routine episiotomies without consent. Annals of nursing and practice, 5 (3). 1102

Ravaldi, C., Skoko, E., Battisti, A., Cericco, M., y Vannacci, A. (2018). Abuse and disrespect in childbirth assistance in Italy: A community-based survey. European journal of obstetrics, gynecology, and reproductive biology, 224, 208-209. https://doi.org/10.1016/j.ejogrb.2018.03.055

Risco, M. I. (2019). Violencia obstétrica durante la atención del parto, puerperio y la percepción de la usuaria (tesis inédita de Licenciatura). Universidad César Vallejo, Trujillo, Perú. Recuperado de http://repositorio.ucv.edu.pe/bitstream/handle/20.500.12692/40428/Risco_VMI.pdf?sequ ence $=5 \&$ isAllowed $=\mathrm{y}$ 
Rodríguez, A. L., y Vargas, Y. (2017). Expresiones del derecho a la salud en la atención pública a mujeres durante el proceso de embarazo en su vinculación con la violencia obstétrica (tesis inédita de Licenciatura). Universidad de Costa Rica, San José, Costa Rica. Recuperado de http://repositorio.sibdi.ucr.ac.cr:8080/jspui/handle/123456789/5911

Sadler, M., Santos, M. J., Ruiz-Berdún, D., Rojas, G. L., Skoko, E., Gillen, P., \& Clausen, J. A. (2016). Moving beyond disrespect and abuse: addressing the structural dimensions of obstetric violence. Reproductive health matters, 24(47), 47-55. https://doi.org/10.1016/j.rhm.2016.04.002

Silal, S. P., Penn-Kekana, L., Harris, B., Birch, S., y McIntyre, D. (2012). Exploring inequalities in access to and use of maternal health services in South Africa. BMC health services research, 12(1), 120. DOI: 10.1186/1472-6963-12-120

Small, R., Yelland, J., Lumley, J., Brown, S., \& Liamputtong, P. (2002). Immigrant women's views about care during labor and birth: an Australian study of Vietnamese, Turkish, and Filipino women. Birth, 29(4), 266-277.

Sobhy, S., Arroyo-Manzano, D., Murugesu, N., Karthikeyan, G., Kumar, V., Kaur, I., ... \& Thangaratinam, S. (2019). Maternal and perinatal mortality and complications associated with caesarean section in low-income and middle-income countries: a systematic review and meta-analysis. The Lancet, 393(10184), 1973-1982. https://doi.org/10.1016/S01406736(18)32386-9

Terán, P., Castellanos, C., González-Blanco, M. y Ramos, D. (2013). Violencia obstétrica: percepción de las usuarias. Revista de Obstetricia y Ginecología de Venezuela, 73(3), 171180. Recuperado de http://ve.scielo.org/scielo.php?script=sci_arttext\&pid=S0048$77322013000300004 \& \operatorname{lng}=e s \&$ tlng=es.

UN General Assembly. Convention on the Elimination of All Forms of Discrimination Against Women. UN General Assembly; 1979 Dec. Recuperado de https://www.ohchr.org/Documents/ProfessionalInterest/cedaw.pdf

United Nations, World Conference on Women. (1996). The Beijing Declaration and the Platform for Action: Fourth World Conference on Women, Beijing, China, 4-15 September 1995. New York: Dept. of Public Information, United Nations. Recuperado de https://www.un.org/en/events/pastevents/pdfs/Beijing_Declaration_and_Platform_for_Ac tion.pdf

Watts, C., Zimmerman, C. (2002). Violence against women: global scope and magnitude. The Lancet, 359(9313), 1232-1237. DOI: https://doi.org/10.1016/S0140-6736(02)08221-1

White Ribbon Alliance (2017). Respectful Maternity Care: The Universal Rights of Childbearing Women. Washington DC: White Ribbon Alliance. Recuperado de: http://whiteribbonalliance.org/wp-content/uploads/2017/11/Final_RMC_Charter.pdf

Wooden, H. E. (1961). Impact of the industrial revolution on hospital maternity care. Nursing forum, 1(1), 90-104.

World Health Organization. (2014). The prevention and elimination of disrespect and abuse during facility-based childbirth: WHO statement [No. WHO/RHR/14.23]. https://www.who.int/reproductivehealth/topics/maternal_perinatal/statement-childbirth/en 
World Health Organization (2002). World Report on Violence and Health. Geneva : OPS

Zeitlin, J., Mohangoo, A., Delnord, M. y Zhang, W. H. (2013). European perinatal health report: health and care of pregnant women and babies in Europe in 2010. 